\title{
Calidad microbiologica de carne bovina en plantas de beneficio
}

\author{
Microbiological quality beef in mills
}

\author{
Martínez P. Cesar, Verhelst S. Adriana \\ Docentes Investigadores, Grupo de investigación CIPTEC, Fundacion Universitaria Tecnologico de \\ Comfenalco, Tecnologia en control de calidad, Cartagena.
}

Recibido 02 Marzo de 2015; aceptado 01 Mayo de 2015

\section{RESUMEN}

Los factores que influyen en la producción de carne y en su calidad son muchos, algunos de ellos corresponden a las propias características del animal como son la especie, la raza, el sexo, el peso y edad de sacrificio. Otros, como la alimentación, el manejo, el transporte a la planta de beneficio, las operaciones de sacrificio y tecnologías de conservación y de prolongación de la vida útil de la carne, son ajenos al animal y pueden ser controlados por el hombre.

El presente documento contiene un análisis general de la información actual acerca de la calidad microbiológica de las carnes y el papel que desempeñan las plantas de beneficio para la producción higiénica de carne bovina y el cumplimiento de la normatividad vigente.

Palabras clave: Carne bovina, calidad microbiológica, plantas de beneficio.

\section{ABSTRACT}

The factors affecting meat production and affect its quality are many, some of them correspond to the characteristics of the animal such as the species, breed, sex, weight and age at slaughter. Others, such as feeding, handling, transport to the slaughterhouse, slaughter operations and maintenance technologies and extending the life of the flesh, are foreign to the animal and can be controlled by man.

This document contains a brief overview of current information about the microbiological quality of meat and the role of processing plants for the hygienic production of beef and compliance with current regulations.

${ }^{*}$ Autor a quien debe dirigirse la correspondencia. E-mail: cmapa@gmail.com

Keywords: Beef, Microbiological quality, Plants benefit. 
Los consumidores de productos de origen animal, exigen y reclaman que dichos productos cumplan las normas de seguridad alimentaria que garanticen su inocuidad, hace que la seguridad alimentaria se considere un problema de estado en todos los países medianamente desarrollados. (Astorga, et al., 2002).

La carne y sus derivados pertenecen al grupo de alimentos de mayor riesgo en salud pública, ya que sus características de composición favorecen la proliferación microbiana, y por consiguiente cualquier deficiencia en sus condiciones de producción, procesamiento, manipulación, conservación, transporte y comercialización puede ocasionar trastornos a la salud del consumidor (CONPES 3376, 2005), (Minor et al., 2002), (ANTAD, 2008) (Castañeda et al., 2001), (Estrada et al., 2004), (Rivera et al., 2004). Los microorganismos que alteran la carne, llegan a ella por infección del animal vivo (contaminación endógena) o por invasión post-mortem (contaminación exógena). Aunque ambas son de gran importancia, la alteración de la carne a consecuencia de la contaminación exógena es la más frecuente, de tal forma que el consumidor puede sufrir graves infecciones o intoxicaciones por el consumo de carne procedente de animales sanos (Restrepo et al., 2011).

El consumo de carne continua, siendo un factor importante en la dieta alimentaria de un buen porcentaje de la población Colombiana, que persevera en sus hábitos de preferencia por las carnes y otros alimentos frescos no refrigerados. El consumo de carnes recién obtenidas dificulta muchas veces el cumplimiento de normas $y$ exigencias que tienden a asegurar que el producto se obtenga en condiciones aceptables desde el punto de vista sanitario; el corto tiempo que media entre el procesamiento y el consumo limita la aparición de signos macroscópicos de alteración con recuentos bacterianos usualmente altos (Bobenrieth et al., 1985).

La susceptibilidad de estos alimentos para ocasionar enfermedades transmitidas por alimentos (ETAs) que no solo afecta significativamente la salud y bienestar de la población, sino que tiene consecuencias económicas para las personas, familias, comunidades, negocios y en general para los países, ya que ocasionan perdidas en la productividad y los ingresos. Mediante el decreto 1500 de 2007 (Decreto 1500, 2007), (Resolución 2905, 2007), el gobierno Colombiano estableció el reglamento técnico a través del cual se crea el Sistema Oficial de Inspección, Vigilancia y Control de la carne, productos cárnicos comestibles y derivados cárnicos destinados para el consumo humano y los requisitos sanitarios y de inocuidad que se deben cumplir en su producción primaria, beneficio, desposte, despiece, procesamiento, almacenamiento, transporte, comercialización, expendio, importación o exportación; con el fin de proteger la salud, la vida y prevenir las practicas que puedan inducir a error a los consumidores. Es evidente que para que lo anterior pueda alcanzar los resultados esperados, se tengan en cuenta las tendencias del sector cárnico como: la producción orgánica de carnes, la producción limpia, la trazabilidad, la denominación de origen, la aplicación de Buenas Prácticas de Producción Animal, de Buenas Prácticas en el uso de Medicamentos Veterinarios, así como las Buenas Prácticas de Manufactura y de sistemas de Gestión de Calidad, los cuales constituyen la garantía para la inocuidad de la carne y el fundamento esencial para el posicionamiento del producto en el mercado nacional e internacional.

\section{Características de la carne como alimento}

La carne es un ingrediente de gran importancia en la alimentación humana. Su alto valor nutricional se debe fundamentalmente a su elevado contenido en proteína de alto valor biológico, pero por otro lado, es uno de los alimentos más perecederos debido a su alta actividad acuosa, composición y $\mathrm{pH}$, lo que favorece la alteración y contaminación microbiana, pudiendo constituir un riesgo para la salud (Sánchez, 2001). 


\section{Contaminación de la carne}

La presencia de agentes patógenos en los productos de origen animal representa un riesgo para la salud publica razón por la cual, en la actualidad, no solo basta con producir la cantidad de proteína necesaria para alimentar a una población cada vez más numerosa, sino que además es indispensable que esos alimentos sean totalmente sanos para los consumidores (Molero et al., 2006). Las operaciones de carnizacion, tienen una vertiente humana de personal y otra técnica de maquinaria y equipos, que afectan directamente a la calidad de la canal y de la carne (Caballero et al., 1997). Las canales y cortes de carne pueden contaminarse debido a agentes físicos, químicos y biológicos en cualquier punto de la cadena productiva, por lo que deben establecerse controles y aplicar las buenas prácticas de manufactura (Mossel, 2003).

La carne que es procesada o manipulada inadecuadamente, puede ser una importante fuente de infecciones o de intoxicaciones alimentarias. Los agentes biológicos más comunes de ETAs en las carnes frescas son salmonella $s p$, stafilococcus aureus, clostridium perfringens y escherichia coli O157:H7. (Dykes, 2004).

Las practicas inadecuadas en el proceso de sacrificio, durante el desangrado, desollado, faenado, eviscerado y despiece de la canal facilitan la contaminación debido al contacto de la carne con suciedad, material fecal y polvo, por lo general, la intensidad con que ocurre la contaminación depende de las normas de higiene y limpieza observadas en la planta de procesado (Untermann, 1989). En las plantas de beneficio una variedad de fuentes contribuye a la contaminación microbiológica durante el proceso, el cual incluye faenamiento, desollado, evisceración, refrigeración y desposte, donde los músculos de los animales son expuestos al ambiente (Jay, 2002), (Heredia et al., 2001), (Kammenou et al., 2003), (Siriken, 2004). Estas fuentes de contaminación pueden ser el aire, agua, suelo, heces, alimento, piel, intestinos, nódulos linfáticos, equipos de procesamiento, utensilios y seres humanos (Koutsoumanis et al., 2004; Godínez et al., 2005; Franco, 2008).
Escherichia coli, pertenece a la flora normal del intestino humano, de ésta se conocen hasta el momento seis serotipos que pueden ser patógenos y causar daño produciendo diferentes cuadros clínicos, entre ellos diarrea, síndrome urémico hemolítico, colitis hemorrágica y cuadros de disentería (Cicuta et al., 2006). Este microorganismo se clasifica en base al grado de patogenicidad y manifestaciones clínicas en: enterotoxigénica (ETEC), enterohemorrágica (EHEC), enteroinvasiva (EIEC), enteropatógena (EPEC), enteroagregativa, (EAEC) y de adherencia difusa (DAEC) (Mossel, 2003), (Dykes, 2004), (Cobbaut et al., 2009), (Gallegos et al., 2009).

Salmonella spp., se considera como "Patógeno Universal" debido a que cuenta con mecanismos de adaptación a diversas condiciones ambientales y por tanto posee una amplia distribución en el medio (Koneman, 2008), ( Vadillo et al., 2002). Se identifican dos tipos de Salmonelosis humanas: las debidas a serotipos estrictamente humanos, que causan síndromes tifoideos con presencia de bacterias en sangre, y las ocasionadas por serotipos ubicuos, que provocan diarrea, vómitos y fiebre (Mossel, 2003), (Kammenou et al., 2003), (Siriken, 2004). Salmonella spp., es responsable de un amplio número de manifestaciones clínicas en los seres humanos como fiebres entéricas, gastroenteritis, bacteriemia, infecciones localizadas y estado de portador crónico. Se transmite por la ingesta de microorganismos presentes en alimentos provenientes de animales infectados, o contaminados con las heces de un animal o persona infectada (Jay, 2002).

Listeria Monocytogenes, el microorganismo ha sido detectado en la tierra, agua, plantas, vegétales, carne, leche y sus derivados, así como en las heces de animales o humanos. No obstante su principal habitad es el suelo y la materia vegetal en descomposición en la cual sobrevive y crece como saprófito (Crespo, 1999). Debido a su amplia distribución este microorganismo tiene muchas oportunidades de contaminar alimentos en distintas partes de la producción alimentaria siendo ésta la vía más frecuente por la que el ser humano adquiere la listeriosis. 
Cría de Animales. La carne puede contaminarse desde la producción primaria. Los peligros más importantes son los químicos y biológicos:

\section{Químicos:}

. Medicamentos veterinarios (antimicrobianos, antiparasitarios, etc.).

. Hormonas y promotores de las producciones.

Contaminantes ambientales: dioxinas, insecticidas, plaguicidas, etc.

\section{Biológicos:}

- Microorganismos: Salmonella, Campilobacter, Listeria, E. coli, etc.

- Parásitos: triquinas, cisticercos, toxoplasmas, etc.

. Priones: enfermedad de las vacas locas.

Manipulación en las plantas de beneficio. La carne fresca puede resultar contaminada por el ambiente de la planta al momento del sacrificio, por lo que los agentes patógenos pueden permanecer en la superficie de la carne o penetrar con algún utensilio en el tejido muscular. (McEvoy et al., 2003). Los puntos o etapas más importantes para el control de la carne son:

El desollado, ya que la piel del animal suele presentarse sucia. Es muy importante que los cuchillos sean de uso exclusivo para esta etapa.

El eviscerado, hay que tener cuidado de ligar bien el esófago y los intestinos para evitar que el contenido intestinal contamine la canal.

La inspección veterinaria oficial, que debe controlar en todo momento las condiciones de trabajo, así como todas las canales de los animales y sus vísceras. Todos los tejidos o canales que entrañen riesgos, o presenten alguna lesión, parásitos o cualquier tipo de anormalidad, se retirarán de la línea y se impedirá su comercialización. En ocasiones, son necesarios análisis complementarios (por ejemplo mal de las vacas locas, residuos medicamentosos, etc.) (ICMSF, 2000),(Caballero, 2008).

El control del agua utilizada en la limpieza de las canales y de las superficies de contacto.

Transporte de Carnes. Para evitar contaminaciones durante el transporte deben tomarse las siguientes medidas:
- Utilizar medios de transporte en buenas condiciones higiénicas (limpias y desinfectadas) y de uso exclusivo para este fin.

- No transportar nunca la carne junto con otros productos que puedan contaminarla.

- Transportar las canales o las piezas colgadas o en recipientes adecuados, evitando el contacto con las paredes o con el suelo del vehículo.

- Realizar el transporte a la temperatura adecuada (refrigeración o congelación).

Almacenamiento, distribución y venta de las carnes. El almacenamiento de las carnes crudas, ya sea en la planta de beneficio, en la industria o en la carnicería debe hacerse en perfectas condiciones de higiene $\mathrm{y}$, por supuesto, sin romper en ningún momento la cadena de frío (Molin et al., 1982). Deben aplicarse las siguientes pautas tanto en el almacenamiento, como la distribución y venta:

. Evitar que los productos estén en contacto directo con el suelo.

. No almacenar las carnes frescas junto con otros productos (productos curados, frutas y verduras, etc.).

. Permitir una circulación de aire adecuada en las cámaras.

- No superar los tiempos de almacenamiento recomendados.

. Aplicar un programa de limpieza y desinfección de las cámaras y locales.

. Vigilar que las cámaras mantengan la temperatura exigida.

- Evitar la contaminación cruzada asociada principalmente a equipos, utensilios y personal manipulador (Godínez et al., 2005).

Acondicionamiento para el procesado. En esta fase, la carne se acondiciona para utilizarla posteriormente, bien en la venta directa al consumidor, bien como materia prima para distintos derivados cárnicos. Entre las operaciones de acondicionamiento se encuentran el despiece, deshuesado, troceado, triturado, picado, descongelación, pelado. El riesgo fundamental en esta fase es que se pueda producir la contaminación microbiana de las carnes o que se puedan multiplicar los microorganismos que ya contienen. 


\section{El Problema de las plantas de beneficio}

En los países desarrollados la mayoría de las enfermedades de origen cárnico son las infecciones y/o intoxicaciones con alimentos, que ocurren cuando la población consume carnes procesadas que se infectan o contaminan durante su preparación (Bobenrieth et al., 1985). Rara vez pasa lo mismo con carne fresca cocinada y los brotes que suceden se identifican con carnes de vacuno, que han sido mantenidas en condiciones deficientes de refrigeración. En los países en desarrollo se verifican más problemas de zoonosis, en especial infecciones parasitarias y diversas septicemias, lo cual a menudo se agrava por las condiciones generales inadecuadas de refrigeración y saneamiento ambiental.

En un estudio realizado en la ciudad de Bogotá por (Guarín, 2008) titulada carne de cuarta categoría para consumidores de cuarta categoría, plantea entre otros aspectos la problemática de las plantas de beneficio en Colombia y señala: "La legislación colombiana que regula el sacrificio y la comercialización de carne, que data de 1982, divide estos establecimientos en cinco clases, dependiendo de la infraestructura con la que cuentan y el tamaño de la población a la que sirven. Las de primera clase tienen las más altas especificaciones técnicas, manejan grandes volúmenes de animales, y pueden producir carne para su consumo en cualquier parte de Colombia o del mundo. Los de segunda clase son un poco menos sofisticados, pero están autorizados para surtir todo el mercado Nacional. Los de las clases III, IV y $\vee$ utilizan técnicas muy rudimentarias, sacrifican pocas reses y sólo se les permite abastecer mercados locales. Los que no cumplen con las mínimas normas de sanidad y que sacrifican menos de una res al día, o los mataderos tipo "planchón", no están incluidos en la clasificación oficial pero representan una fracción importante del sacrificio de animales en Colombia, sobre todo en pequeños municipios y áreas rurales. En muchos de estos planchones, toda la operación de corte y desposte se hace sobre la propia piel del animal muerto (Koutsomanis, 2004).

En el país se tiene como propósito la adopción de políticas sanitarias drásticas en cuanto al mantenimiento de las condiciones higiénicosanitarias de los frigoríficos (Franco, 2008).

Los problemas en las plantas de beneficios y las plantas procesadoras de productos cárnicos se suscitan a raíz de factores como:

- Poco desarrollo económico del País, imposibilitando al estado para hacerse cargo de las elevadas inversiones de capital para las plantas físicas de sacrificio y desposte de animales de la especie bovina, así como del procesamiento moderno y control sanitario de las mismas.

- Función que desempeñan las plantas de beneficio como fuentes de financiamiento municipal, estatal o privado sin que se efectúen reinversiones para mantenimiento $y$ modernización, así como la contratación de personal calificado.

- Bajo nivel de educación del personal vinculado con la explotación, procesamiento, comercialización y distribución de los productos.

- Planificación y ejecución de programas de educación y adiestramiento, por parte de profesionales y técnicos, desvinculados de la realidad de recursos.

- Proliferación de recomendaciones de expertos externos o de funcionarios de instituciones sobre perfeccionamiento de plantas procesadoras, que resultan inaplicables en la realidad.

Todos estos factores plantean la necesidad de buscar otros criterios para abordar el problema y hallar soluciones efectivas acordes con la realidad del país.

\section{Análisis microbiológico de la carne}

La carne en su proceso de obtención, se convierte en un alimento con alta probabilidad de generar enfermedad en el consumidor por los microorganismos patógenos que llegan a ella, también se contamina por microorganismos saprofitos, que van a alterarla, a menos que se mantenga refrigerada o congelada (Marsden, et al., 1994). La importancia de las bacterias en relación a la carne, reside principalmente en que ellas están 
íntimamente ligadas al proceso de infección e intoxicación alimentaria.

\section{Microorganismos indicadores}

La difícil detección de patógenos debido a su baja concentración y desigual distribución en las muestras de alimentos han requerido el uso de "microorganismos indicadores" (Schaffner et al., 2004). Estos microorganismos y/o sus productos metabólicos cuya presencia en los alimentos, en niveles determinados, pueden ser empleados para reflejar su calidad microbiológica (Jay 2000), (Pascual 2000). Los indicadores de calidad microbiana son microorganismos causantes de alteración cuyo número creciente da como resultado la pérdida de calidad del producto (Schaffner et al., 2004). Los indicadores también pueden revelar fallas en el proceso que podría permitir que un alimento este contaminado o la multiplicación de un patógeno a niveles peligrosos (Schaffner et al., 2004). Al respecto, los indicadores de inocuidad comúnmente usados son: $S$. aureus, estreptococos fecales, entero bacterias, anaerobios sulfito reductores, esporas de aerobios $y$ anaerobios, mohos, levaduras y el recuento de aerobios mesófilos. Las especies que habitualmente se encuentran en las carnes y producen disminución de su vida útil, reflejado en alteraciones organolépticas (olor, sabor, color y textura) pertenecen a los géneros: Pseudomonas, Achromobacter, Acitenobacter, Streptococcus, Micrococcus, Sarcina, Leuconostoc, Flavobacterium, Proteus, Escherichia, Bacillus, Clostridium, Chromobacterium, Streptomyces, levaduras y mohos. En carnes almacenadas a baja temperatura en condiciones aeróbicas, Pseudomonas spp es considerado el principal organismo de deterioro, mientras que bacterias Gram-positivas, como bacterias formadoras de esporas y Brochotrix thermosphacta, son responsables del deterioro en carne envasada al vacío y otras condiciones de envasado en atmosfera modificada. Hongos y levaduras raramente contribuye al deterioro de carne fresca, $y$, generalmente, puede ser detectado solo durante almacenaje extendido y cuando la superficie de la carne se seca, lo cual limita el crecimiento microbiano y permite a las levaduras de crecimiento más lento a dominar (Koutsomanis et al., 2004). Además, en la carne se puede encontrar gérmenes dañinos para la salud humana, procedentes de animales enfermos o de malas manipulaciones durante su preparación, estos pueden ser: Salmonella spp., S. aureus, Yersinia enterocolítica, Campylobacter jejuni, E. coli 0157:H7, Listeria monocytogenes, $\mathrm{Cl}$. perfringens, Clostridium botulinum (Pascual 2000, Mossel 2003, Koutsomanis et al., 2004). Salmonella spp., E. coli patógeno y Campylobacter son de origen entérico y considerados patógenos comunes de enfermedad alimentaria en carnes. Estos últimos, tienen la facultad de crecer en amplios rangos de temperatura que pueden ir desde 0 a $45^{\circ} \mathrm{C}$ aproximadamente (Jay, 2000).

Para mantener una adecuada calidad higiénica en la carne se hace necesario que se controle de forma periódica tanto los manipuladores, las canales, el agua, como los utensilios utilizados durante el faenado del animal (Cicuta et al., 2006).

\section{CONCLUSIONES}

En las plantas de beneficio una variedad de fuentes contribuye a la contaminación microbiológica durante las etapas de procesamiento, donde los músculos de los animales son expuestos al ambiente. Estas fuentes de contaminación pueden ser el aire, agua, suelo, heces, alimento, piel, intestinos, nódulos linfáticos, equipos de procesamiento, utensilios y seres humanos. Posteriormente, la contaminación prosigue durante el transporte de las canales o cortes de carne a los lugares de venta, donde puede ocurrir contaminación durante su almacenamiento si las condiciones higiénicas son desfavorables. El modo, el número y clase de los microorganismos presentes en la carne bovina se ve influenciado básicamente por los siguientes factores:

- Ambiente general en que la carne ha sido originalmente obtenida. 
- Su calidad microbiológica en estado fresco o no elaborado.

- Las condiciones sanitarias en que ha sido manipulada.

- Las condiciones en que se haya almacenado, transportado y conservado.

La carne que es procesada o manipulada inadecuadamente, puede ser una importante fuente de infecciones o de intoxicaciones alimentarias. Los agentes más comunes de ETA en las carnes frescas son Salmonella spp., Staphylococcus aureus, Clostridium perfringens y Escherichia coli O157:H7, causante de colitis Entero- Hemorrágica.
La presencia de agentes patógenos en carnes y productos cárnicos, representa un riesgo para la salud pública, razón por la cual, en la actualidad no solo basta con producir la cantidad de proteína necesaria para alimentar una población cada vez más numerosa, sino que además es indispensable que dichos productos sean totalmente sanos para los consumidores.

La aplicación de medidas que permitan reducir la contaminación de la carne en las plantas de beneficio y puntos de distribución, contribuirán a mejorar su calidad microbiológica y garantizar la inocuidad alimentaria, permitiendo el crecimiento del sector agropecuario, base de nuestra economía.

\section{REFERENCIAS BIBLIOGRÁFICAS}

Astorga, R.I., Usera, M.A., Maldonado A., Arenas A., Tarradas C., Luque I., Pérez J., Perea A. 2002. Marcadores epidemiológicos y sensibilidad antimicrobiana en Salmonellas de origen animal. Laboratorio Veterinario Avedila, 21: pp. 2-6.

ANTAD. Asociación Nacional de Tiendas de Autoservicio y Departamentales. Recuperado el 15 Sep, 2008, Disponible en http:// .antad.org.mx ICasociados.html. 2002. Consultada , Abril 2014

Caballero T. A. 2008. Temas de Higiene de los alimentos. Ed. Ciencias Médicas, La Habana. ISBN. 978-959-212-363-2.

Castañeda, PE, Diaz AE, Hernandez AL, Jaramillo ACJ. Identification and typing of Yersinia enterocolitica biotypes and serotypes isolated. 2001; Revista Saude Publica (35):380- 384.

Cicuta M, Deza N, Roibón W, Benitez M, Ramírez G, Arzú R. Escherichia coli productor de toxina Shiga en reses bovinas y carne molida de Corrientes, Argentina. Revista Argen Vet.:(17):20-25.

Comisión Internacional de Especificaciones Microbiologicas para los Alimentos (ICMSF), El sistema de análisis de riesgos y puntos críticos, su aplicación a la industria de alimentos. 2000. (Recuperado, 10 de mayo de 2011).
Cobbaut K, Berkvens D, Houf K, de Deken R, de Zutter L. Escherichia coli $\mathrm{O} 157$ prevalence in different cattle farm types and identification of potential risk factors. J Food Prot 2009; 72:1848-1853.

Crespo, M., "Aislamiento de Listeria monocytogenes en un hospital de tercer nivel" 1999, Disponible en http://www.monografias.com/trabajos905/aislamiento-listeriamonocytogene/aislamiento-listeria monocytogene.shtmautorizada por: Corporación Editora Médica del Valle, Colombia, Publicación original: Colombia Médica. Consultada abril de 2013.

Decreto 1500. Ministerio de la protección Social. República de Colombia. de 2007

Dykes G. 2004. Escherichia coli O157:H7. In: Jensen W, C Devine, M Dikeman (eds). Encyclopedia of Meat Sciences Series Three-Volume Set: Vol 1-4. 1st ed. Elsevier, New Zealand, Pp 781-785.

Documento CONPES 3376. Política Sanitaria y de Inocuidad para las cadenas de la carne bovina y de la leche. Consejo Nacional de Política Económica y Social. República de Colombia. Departamento Nacional de Planeación. Bogotá, D.C., Septiembre de 2005. Disponible en http://www.ica.gov.co/getattachment/3b31038a-72ba-40f9a34d-cecd89015890/2010cp3676.aspx. consultado Mayo de 2014. 
Estrada GT, Lopez SC, Zamarripa AB, Thompson MR, Gutierrez $\mathrm{CL}$, Mancera MA, Escobar GA. Prevalence of Escherichia coli and Salmonella spp. in street-vended food of open markets (tianguis) and general hygienic and trading practices in Mexico City. Epidemiol Infect 2004;(132):1181-1184.

Franco, J.,Romero, M. \& Taborda, G. . Determinación de niveles residuales de tetra- ciclina en canales bovinas por la técnica de ELISA en el frigorífico Friogan (La Dorada). Biosalud: 2008. Revista de Ciencias Básicas, 7(1), 47-55.

Gallegos M, Morales A, Álvarez G, Vásquez J, Morales L, Martínez I, Maldonado J. Caracterización de aislados de Escherichia coli $\mathrm{O} 157: \mathrm{H} 7$ en canales de bovinos y porcinos mediante PCR. Rev Cient FCV-LUZ 2009; 2:139-146.

Godínez, G.; Reyes,J.;Zúñiga,A.; Sánchez, I.; Castro, J.; Román,A., et al. Condiciones microbiológicas en cuatro rastros municipales del estado de Hidalgo. 2005. Disponible en http://www.respyn.uanl.mx/especiales/2005/ee-13-

2005/documentos/CNA47.pdf. consultado Mayo 2014.

Guarin, A. Carne de cuarta para consumidores de cuarta. 2008. Revista de estudios sociales N²9. ISSN 0123-885X. Pp 104119. processing plants in the United States. J Food Prot 2004;(67):295-302.

Heredia N, S García, G Rojas, L Salazar. 2001. Microbiological condition of ground meat retailed in Monterrey, Mexico. J Food Protect 64, 1249-1251.

Kammenou M, J Metaxopoulos, E Drosinos. 2003. Microbiological quality of minced beef from butcher shops and supermarkets. Ital J Food Sci 1, 95-104.

Jay J. Indicadores de la calidad e inocuidad microbianas de los alimentos. En: Jay J (ed). Microbiología moderna de los alimentos. 4a ed. Acribia, Zaragoza, España, 2002 p 363-379.

Koutsomanis K, J Sofos. 2004. Microbial contamination. In: Jensen W, C Devine, M Dikeman (eds). Encyclopedia of Meat Sciences Series Three-Volume Set: Vol 1-4. 1st ed. Elsevier, New Zealand, Pp 727-736.

Koneman E. Diagnóstico Microbiológico. 6a ed. Argentina, Panamericana, 2008. p. 238-248.
Mattar S, Vásquez E. Escherichia coli O157:H7 infection in Colombia. Emerging Infec Dis 1998;4:126-127.

Mcevoy Jm, Doherty Am, Sheridan Jj, Thomson-Carter Fm, Garvey $P$, Mcguire $L$ et al. The prevalence and spread of Escherichia coli O157:H7 at a commercial beef abattoir. J Appl Microbiol 2003; 95:256-266.

Minor-pérez, h.; Ponce, e.; Macias, s.; Guerrero, I. Conservación de la carne fresca de cerdo por fer- mentación láctica: Efecto sobre el color, la textura y la formación de los ácidos grasos libres. 2002. Revista. Mex. Ing. Química (1) :73-80.

Molin, G \& Fernstrom, A.. Numerical taxonomy of psychrotrophic Pseudomonads. 1982 Journal of general Microbiology. (128) 1249-1264.

Mossel D. Valores de referencia o normas microbiológica. En: Mossel D (ed). Microbiología moderna de los alimentos. $2^{\mathrm{a}}$ ed. Zaragoza, España, Acribia, 2003 p 512-513.

Molero G., Perez A. M., Mavarez m., Sánchez A., Ascanio E. y Oviedo M. Residuos de enrofloxacina en tejido hepático y muscular de pollos beneficiados en el municipio San Francisco, Zulia, Venezuela. 2006 Revista Científica FCV-LUZ, I. (XVI) (6) pp. 629-633.

Molero G., Tarradas C., Ramírez I., Gallardo F., Mmontiel M. Aislamiento e identificación de Listeria monocytogenes durante el proceso de beneficio de pollos en plantas beneficiadoras en el estado Zulia, Venezuela. 2010. Revista Ciencia. 18(2), pp. 108-114.

Pérez C., Riviera S., Pirela A., Rincón H., Mavarez Y., Román R. Aislamiento de Salmonella en carne de aves y evaluación de la efectividad de diferentes medios enriquecidos y selectivos. 2004 Revista Científica 14 (2) Maracaibo abr.

Resolución 2905 de 2007. Ministerio de la protección social. República de Colombia.

Sánchez-Arjona M; Sánchez-Arjona S. "Sistemas de calidad en productos alimentarios de origen animal: caso de Ternera de Navarra" Escuela de Ingenieros. Universidad de Navarra. 2001. 
Restrepo D, Arango C, Restrepo R, Amezquita A. Industria de carnes. Universidad Nacional de Colombia sede Medellín. Julio de 2011. ISBN en trámite.

Bobenrieth R, Beltrán F, Arenas A. Saneamiento de Mataderos de Bovinos, Ovinos y Porcinos. Oficina Sanitaria Panamericana. 1985.

Rivera BM, Shackelford SD, Arthur TM, Westmoreland KE, Bellinger G, Rossman M, Reagan JO, Koohmaraie M. Prevalence of Escherichia coli O157:H7, Listeria monocytogenes, and Salmonella in two geographically distant commercial beef.

Siriken B. The microbiological quality of ground beef in Aydin and Afyon Provinces, Turkey. 2004. Revue Méd Vét (155), 632636.

Untermann F. Hygiene in meat production and processing. Fleischwirtschaft-1989; 69:1026-1029.

Vadillo S, Piriz S, Mateos E. Manual de microbiología veterinaria. Ed. McGraw Hill. Madrid 2002; p. 327-338. 